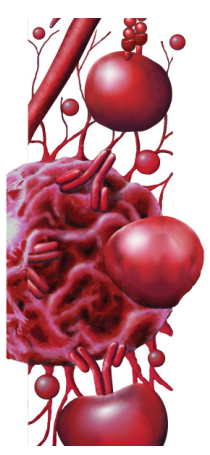

\title{
Modulatory effects of bortezomib on host immune cell functions
}

\begin{abstract}
Bortezomib is an inhibitor of the ubiquitin-proteasome proteolytic pathway responsible for intracellular protein turnover. Cellular proteins controlled by this pathway represent a diverse group of potential therapeutic targets, particularly in cancer cells, which exploit this proteasomal pathway to promote their growth and diminish apoptosis. Along with inhibiting the proteasome and thus sensitizing tumor cells to apoptosis, bortezomib may also have multiple effects on the host immune responses. This review summarizes the effects that bortezomib may play on immune cell subsets in various disease states in modifying lymphocyte receptors, ligands, the expression of various cytokines and chemokines and their downstream signaling. We also propose steps that can be taken to refine combinatorial strategies that include bortezomib to improve current immunotherapeutic approaches.
\end{abstract}

Keywords: B cells $\bullet$ bortezomib $\bullet$ chemokine $\bullet$ cytokine $\bullet$ dendritic cells $\bullet$ immunity $\bullet$ inflammation $\bullet$ ligands $\bullet$ lymphocyte receptors $\bullet$ NK cells $\bullet$ T cells

\section{The ubiquitin-proteasome system $\&$ its physiologic relevance}

The proteasome is a high-molecular-weight multicatalytic protease that degrades intracellular ubiquitinated proteins. It plays a vital role in degrading most short-lived intracellular proteins that regulate a wide spectrum of events in the cell such as cell cycle, cell differentiation, cell death, DNA repair, transcription, signal transduction, morphogenesis, metabolism and antigen (Ag) presentation. The proteasome functions as the main nonlysosomal endoprotease enzyme complex within the nucleus and cytoplasm of all eukaryotic cells in order to eliminate damaged and abnormal proteins to ensure intracellular protein quality [1].

The proteasome is a cylindrical shaped, large hollow 26S enzymatic complex assembled from at least 66 proteins via the aid of multiple chaperone proteins [2-4]. It is composed of two $11 \mathrm{~S}$ or $19 \mathrm{~S}$ regulatory units at either end of the $20 \mathrm{~S}$ catalytic core. The $20 \mathrm{~S}$ catalytic core is arranged into a stack of 47 subunit rings, where the bottom and top rings are formed by seven polypeptides ( $\alpha$-subunits), and the two inner rings are composed of seven $\beta$-subunits. The interaction between the 19S (11S) and 20S particles are driven by polyubiquitination. Once the polyubiquitinated protein is identified by $19 S$, hydrolases unfold the substrate protein so it may enter the narrow gate of the $20 \mathrm{~S}$ catalytic core particle and be degraded [1]. In physiological conditions, inhibition of the proteasome prevents aberrant and misfolded proteins from being degraded. This can build up intracellular endoplasmic reticular stress and lead to cellular apoptosis.

\section{Proteasome inhibition as a therapeutic modality in cancer}

The proteasome is an exciting target for the development of novel anticancer therapies [5]. The intracellular accumulation of Ub-protein conjugates following inhibition of the proteasome triggers cellular apoptosis, with tumor cells being more susceptible to proteasome inhibition than nonmalignant cells. Bortezomib (Velcade, PS-341), a therapeutic drug in
Samuel Troy Pellom Jr, 1,3 Duafalia Fred Dudimah', Menaka Chanu Thounaojam", Thomas Joseph Sayers ${ }^{4,5}$ \& Anil Shanker*,1,2,6

'Department of Biochemistry \& Cancer Biology, School of Medicine, Meharry Medical College, Nashville, TN 37208, USA

${ }^{2}$ School of Graduate Studies \& Research, Meharry Medical College, Nashville, TN 37208, USA

${ }^{3}$ Department of Microbiology \& Immunology, School of Medicine, Meharry Medical College, Nashville, TN 37208, USA

${ }^{4}$ Cancer \& Inflammation Program, National Cancer Institute, Frederick, MD 21702, USA

${ }^{5}$ Basic Sciences Program, Leidos Biomedical Research, Inc., Frederick, MD 21702, USA

${ }^{6}$ Host-Tumor Interactions Research

Program, Vanderbilt-Ingram

Comprehensive Cancer Center,

Vanderbilt University, Nashville,

TN 37232, USA

*Author for correspondence:

Tel.: +1 6153276460

Fax: + 16153276442

ashanker@mmc.edu 
cancer management, is the first therapeutic proteasome inhibitor to be tested in humans. In 2003, bortezomib was approved by the US FDA for the treatment of multiple myeloma (MM), and in 2006 for mantle cell lymphoma [6,7]. In MM, complete clinical responses have been obtained in patients with otherwise refractory or rapidly advancing disease. Trials are currently ongoing with bortezomib for the treatment of some solid cancers such as non-small-cell lung carcinoma [6].

Bortezomib is a dipeptide boronate proteasome inhibitor whose boronic acid moiety has been shown to exert inhibitory effects on the chymo-trypsin-like subunits ( $\beta 5 \mathrm{c}$ and $\beta 5 \mathrm{i}$ ) of the $20 \mathrm{~S}$ core of the proteasome. The boronic acid moiety of bortezomib with its transient (9-15 h half-life) and reversible inhibition of the proteasome set it apart from other proteasome inhibitors, and make it more suitable as a therapeutic agent. Unlike other nonboronic acid proteasome inhibitors, the boronic acid moiety of bortezomib prevents it from being transported out of the cell by the multidrug resistance system.

Nonetheless, concerns over the inhibitory side effects of bortezomib on various immune functions have been cited [8-13]. By contrast, there are also numerous reports indicating that bortezomib, directly or indirectly, may augment immune effector functions under some circumstances [14-21]. This review compares the positive and negative effects of bortezomib on different immune cells to assess the possibility of harnessing bortezomib in various pathophysiologic conditions including cancer.

\section{Host responsiveness to bortezomib treatment \\ Modulatory effects of bortezomib on T cells}

Negative effects of bortezomib on immune cells have been reported in the literature, and chief among these are signs of lymphopenia in a wide range (11-58\%) of Phase II clinical trial leukemia patients following bortezomib treatment $[7,22]$. A higher incidence in varicella zoster virus reactivation has also been reported due to a decrease in CD4 T-cell populations following treatment with bortezomib $[10,23]$. Not only does bortezomib affect the number of CD4 T cells, it has also been shown to reduce their production of Th1 cytokines $[24,25]$. The observed bortezomib-induced reduction in the effector function of $\mathrm{T}$ cells can be partially attributed to the increase of regulatory $\mathrm{T}$ cell (Treg) populations [25]. The effect of bortezomib on $\mathrm{T}$ cells has been shown to be linked to their activation status as resting $T$ cells were less susceptible to the effects of bortezomib when compared with activated $T$ cells [24]. This observation is, however, debatable in view of the more recent findings. Another mechanism that may account for the decrease in CD4 T cells and lessened T cell cytotoxicity may be attributed to bortezomib's effect on Ag presentation by dendritic cells (DCs). However, some of the inhibitory effects on immune cell function ascribed to bortezomib based on in vitro studies should be viewed with caution. In some studies, the negative side effects may be attributed to the high concentration of bortezomib that were used. Concentrations higher than $20 \mathrm{nM}$ have been observed to be cytotoxic to cells over a 48-72-h period and some of these reports use concentrations as high as $100 \mathrm{nM}$ in short-term assays. The administration of lower doses of bortezomib in vivo may provide therapeutic benefit under some circumstances in the apparent absence of major side effects [26].

Bortezomib enhanced Ag-specific cytotoxic T-cell responses against immune-resistant cancer cells generated by STAT3-ablated DCs [27]. Also, bortezomib could restore MART-1 Ag expression on human melanoma cells to sensitize them to specific CTLs in vitro [28]. It is worth noting that bortezomib inhibits inducible NF- $\mathrm{KB}$ activity, but can activate constitutive NF- $\kappa \mathrm{B}$ activity by triggering phosphorylation of IкB kinase and its upstream receptor-interacting protein RIP2, thereby enhancing cytotoxicity in tumor cells [29]. Our recent data also suggest that bortezomib sustained FasL-mediated T-cell cytotoxicity against tumors by stabilizing expression of IL-2 receptor $\alpha$ chain and T-cell receptor $\mathrm{CD} 3 \zeta$ in T-cells of tumor-bearing mice.

\section{Effects of bortezomib on B cells}

$\mathrm{B}$ cells play a vital role in antibody $(\mathrm{Ab})$ mediated immune responses. The normal function of $\mathrm{B}$-cells has been reported to be impaired upon bortezomib treatment $[13,30]$. These studies have shown that activated $\mathrm{B}$ cells are most susceptible to bortezomib, which renders these cells less capable of initiating Ab-mediated responses [13,30-31]. The decrease in Ab secretion is thought to be associated with the bortezomib-induced enhancement of apoptosis of Ab-secreting cells such as plasma cells or memory B cells [31]. Proliferation of activated B cells is significantly reduced in a dosedependent manner within seven days of bortezomib treatment. In a study of the effects of bortezomib on activated B-cell function following polyclonal stimulation, it was observed that a low dose $(2-3 \mathrm{nM})$ bortezomib inhibited the secretion of IgM and IgG. In the same study, these activated B cells showed a dosedependent increase in apoptosis in response to bortezomib, which may have accounted for the decreased proliferation and reduced immunoglobulin production [13]. Thus, bortezomib treatment can result in a significant impairment of B-cell function, thereby ren- 
dering these cells less capable of initiating Ab-mediated responses.

\section{Effects of bortezomib on DCs}

There are conflicting findings concerning the effect of proteasome inhibitors on the function of DCs. The reported effects of bortezomib on DCs are far reaching, and may result in a reduction of cytokine production, increased apoptosis and loss of Ag-presenting function [22,26,32-34]. Specifically, bortezomib-induced apoptosis is mediated through upregulation of Bax in DCs [32]. The Ag-presenting function of DCs has been shown to be impaired by bortezomib through an inhibition of costimulatory molecule expression. Bortezomibinduced loss of migratory abilities of DCs, coupled with its ability to desensitize DCs to immunostimulation by TNF- $\alpha$ and lipopolysaccharide (LPS) are other contributory factors that could account for a reduction of $\mathrm{Ag}$ presentation [26,34]. Furthermore, bortezomib reduces DC-induced allogenic T-cell proliferation while concurrently inhibiting the expression of DC maturation markers [9]. Plasmacytoid DCs (pDCs) are a subset of DCs that are thought to be essential players in antiviral immune responses by the production of IFN- $\alpha[35,36]$. Among all immune cells studied, pDCs were found to be the most susceptible to the killing effects of bortezomib at physiologically relevant concentrations [37-39]. Other reported negative effects of bortezomib on $\mathrm{pDC}$ function include induction of apoptosis through the inhibition of XBP1, which is essential for development of pDCs and other plasma cells [12,37,40-41]. The trafficking of TLR9 from the ER to the endolysosomes and cytokine production in DCs has also been shown to be suppressed by bortezomib [37]. In another subpopulation of proinflammatory myeloid human DCs known as 6-Sulfo-LacNAc (slan) DCs, differing from other blood DC subsets in their phenotype 6-Sulfo-LacNAc ${ }^{+} \mathrm{CD} 1 \mathrm{c}^{-}$

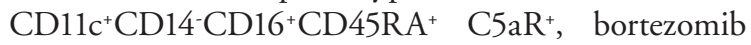
can inhibit their maturation, cytokine production and their capacity to activate natural killer (NK) cells [32-34].

Interestingly, while bortezomib has been shown to have negative effects on DCs, it has also been demonstrated that by promoting the exposure of heat shock proteins $60 / 90$ on dying tumor cells bortezomib can increase their phagocytosis by DCs [21]. In addition, DCs that were treated with bortezomib in vitro showed increased maturation, which resulted in potent immunemediated antitumor effects [16]. It is also reported that bortezomib induces apoptosis in immature DCs, but to a much lesser extent in mature DCs [42].

\section{Effects of bortezomib on NK cells}

NK cells are large granular lymphocytes that constitute a key cellular subset of the innate immune system.
Bortezomib has been shown to decrease the expression of HLA class-I molecules in a time- and dose-dependent manner on MM cells [20], thereby sensitizing them to both allogenic and autologous NK cell mediated lysis. This study demonstrated therapeutic implications of adoptively transferred allogenic and autologous NK cells for NK-sensitive malignancies. Bortezomib can also sensitize tumor cells to NK cells by up-regulating the expression of TNF receptor apoptosis-related protein $\mathrm{p} 55$ (TNFRp55), receptor-interacting protein RIP and death receptor (DR) 3, DR4 and DR5 [14]. Bortezomib sensitized three breast cancer cell lines to NK cell-mediated killing in a long-term in vitro tumor outgrowth assay. While bortezomib could be cytotoxic to NK-92 cells in vitro, it was observed that bortezomib treatment in vivo did not decrease NK-92 function.

Hallet et al. provided an insight into the mechanisms underlying NK cell antitumor effector function against cancer. Preincubation of leukemia cells with bortezomib had no effect on short-term NK cell killing or granule exocytosis pathway [17]. Long-term tumor outgrowth assays allowed for the detection of tumor cell killing following bortezomib treatment by perforin-deficient NK cells, which was dependent on both TRAIL and FasL on NK cells, correlating with an increase in tumor cell Fas and DR-5 expression [17]. These observations were confirmed in the context of human prostate cancer cells, which showed sensitivity to NK cells following bortezomib treatment in longterm assays. Treatment with bortezomib caused prostate cancer DU145 cells to upregulate Fas as well as DR5 and conversely, downregulate the MHC-I molecules on their surface [43]. In turn, therapeutic strategy involving bortezomib may be more effective in patients with androgen-insensitive prostate cancer. Thus, bortezomib treatment can sensitize tumor cells to cyotoxic lymphocyte effector pathways; suggesting that the combination of bortezomib with adoptive cell therapy may result in increased antitumor efficacy if an optimal dosing and timing of bortezomib administration is used.

Bortezomib was also shown to upregulate TRAIL receptor expression on primitive $\mathrm{CD} 34^{+}$quiescent chronic myeloid leukemia (CML) cells, known to be more resistant to the effects of tyrosine kinase inhibitors than their differentiated cycling CML counterparts, and thus increase their susceptibility to cytotoxicity by in vitro expanded donor NK cells [44]. This study suggests that donor-derived NK cell-mediated graft-versus-leukemia effect can possibly be improved by using bortezomib to sensitize quiescent $\mathrm{CD} 34^{+}$ CML cells to NK cell cytotoxicity following stem cell transplantation. Bortezomib therapy has also been investigated in acute myeloid leukemia (AML) [45]. A 
higher sensitivity of CD34- AML cells to $20 \mathrm{nM}$ treatment of bortezomib in comparison with the CD34 ${ }^{+}$ AML cells was suggested to be due to differences in NF- $\kappa \mathrm{B}$ activation and accumulation of MCL-1, an induced myeloid leukemia cell differentiation protein that regulates apoptosis, in $\mathrm{CD} 34^{+}$versus CD34AML cells. Thus, combining bortezomib with specific NF- $\mathrm{KB}$ or MCL-1 inhibitors may potentially improve targeting of the leukemic stem cells.

The combination therapy of bortezomib with infusions of death ligand, such as TRAIL-expressing lymphocytes may reduce the relapse in CML and AML patients who require treatment by stem cell transplantation. Taken altogether, bortezomib can play a beneficial role in enhancing innate immune effector function by sensitizing various cancer cells to NK cell-mediated lysis.

\section{Effects of bortezomib on signaling through NK} cell-activating receptors

With bortezomib having shown potential to enhance NK cell immunity, we will now review how bortezomib influences interactions between NK cell receptors and their various ligands on cancer cells. In a study by Soriani et al., myeloma cells treated with low doses of bortezomib upregulated expression of NK group 2, member D (NKG2D) and DNAM-1 ligands. Bortezomib treatment of MM cells increased NK cell degranulation response, due to interaction between the NK receptors NKG2D and DNAM-1 and the increased levels of their cognate ligands on the myeloma cells [46]. Analogous data were also observed using ex vivo primary plasma cells obtained from MM patients. In addition, bortezomib treatment of these MM cells was associated with arrest in the G2 phase of the cell cycle and cell senescence. Overall, the study identified a common bortezomib-induced pathway that can trigger the upregulation of different NK cell-activating ligands on cancer cells, and proposed that NK cells can represent an immunosurveillance mechanism targeting cells undergoing stress-induced senescent programs.

A similar study by Armeanu et al. demonstrated that low dose bortezomib (2-8 nM) could enhance NK cell antitumor activity in a hepatocellular carcinoma (HCC) model. This study provided insight on the tumor-associated ligands that engage and activate NK cell receptors such as NKG2DL and UL-16-binding protein (ULBP) families, which potently stimulate NK cell responses [15]. Bortezomib treatment increased levels of NK cell-activating receptor ligands, MHC class-I chain-related gene $\mathrm{A}(M I C A)$ and $M I C B$ on hepatoma cells, thus stimulating cytotoxicity and IFN- $\gamma$ production by cocultured NK cells. Interestingly, low-dose bortezomib treatment did not alter NKG2DL expres- sion, nor induce cell death in primary human hepatocytes. Therefore, bortezomib treatment mediates a dual antitumor effect in HCC, by inhibiting tumor cell proliferation and priming hepatoma cells for NK cell cytotoxicity. Thus, patients with HCC may benefit from bortezomib treatment combined with immunotherapeutic strategies such as adoptive NK cell transfer, taking advantage of the enhanced NKG2D-mediated antitumor activity [15]. Also, in a head and neck squamous cell carcinoma (HNSCC) model, bortezomib treatment led to an upregulation of stress-activated NKG2D ligand ULBP1 on tumor cells. HNSCC cells usually express MICA, MICB, ULBP2 and ULBP3, but are uniformly negative for cell surface ULBP1. Butler et al. observed that proteasome inhibition via bortezomib dramatically and specifically upregulated HNSCC ULBP1 mRNA and surface protein levels [47].

In a primary acute lymphoblastic leukemia (ALL) model, bortezomib induced NKG2D ligands and sensitized these ALL cells to NK cell recognition. ALL had previously been considered resistant to NK cell lysis [48]. Jardine et al. demonstrated that bortezomib can upregulate NK-activating ligands on a B-cell ALL cell line and on adult primary B-cell ALL samples [48]. Bortezomib-treated ALL cells triggered higher levels of NK degranulation by CD107a expression, and this effect was dependent on signaling through the NK cell-activating receptor NKG2D. This suggests that bortezomib may also have clinical utility in sensitizing ALL to NK-mediated lysis in vivo. Altogether, a number of studies have determined that bortezomib given at concentrations $<10 \mathrm{nM}$ increased multiple ligands such as NKG2DL, DNAM-1 and MICA/B on cancer cells, resulting in NK cell activation, enhanced cytotoxicity and increased IFN- $\gamma$ production. Therefore, for immunotherapeutic strategies with NK cells, it may prove more beneficial to adoptively transfer NK cells following bortezomib treatment to promote an enhanced eradication of the tumor cells.

A combination of bortezomib with exogenous cytokine treatments may further enhance the cytotoxic effects of NK cells against cancer cells. In fact, bortezomib can maximize the apoptotic potential of cytokines in tumors [49]. A study by Wang et al. investigated if the combination of bortezomib and immune therapy with IL-12 could produce increased antitumor effects compared with therapy with the individual agents. The study showed IL-12 treatment alone produced a mild decrease in tumor volume compared with control; bortezomib alone was observed to have extensive antitumor effects with approximately 65 and $60 \%$ reduction in tumor volume after 15 and 21 days of therapy, respectively. However, at the same time points a significant difference was seen in comparing 
bortezomib treatment alone to a combination therapy of bortezomib and IL-12 which generated approximately a 75 and $84 \%$ reduction in tumor volume, respectively [50]. Beneficial effects of the combination could be explained by the fact that IL-12 is involved in the stimulation and production of IFN- $\gamma$ and TNF- $\alpha$ from T-cells and NK cells, and reduces IL-4-mediated suppression of IFN- $\gamma$. Moreover, supplementation with IL-2, which stimulates the expression of IL-12 receptors, IL-12R- $\beta 1$ and IL-12R- $\beta 2$, can improve NK cell functional response by enhancing IFN- $\gamma$ production and killing of target cells. In conclusion, bortezomib combined with IL-2/IL-12 therapy had a substantial antitumor effect relative to monotherapy with the individual agents alone; further suggesting, the combination therapy including cytokines with bortezomib may result in an effective tumor therapeutic approach.

Berg et al. developed a method to generate $\mathrm{TRAIL}^{+}$ $\mathrm{FasL}^{+} \mathrm{NKG}^{2} \mathrm{D}^{+}$clinical grade NK cells following culture with irradiated Epstein-Barr virus (EBV)transformed lymphoblastoid cells. This ex vivo NK cell expansion technique played a role in increasing the expression of activating receptors and DR ligands resulting in superior cytotoxicity against tumor cells. When these expanded NK cells were cocultured with K562 and renal adenocarcinoma tumor targets, they secreted significantly higher levels of soluble FasL, IFN- $\gamma$, GM-CSF, TNF- $\alpha$, MIP- $1 \alpha$ and MIP- $1 \beta$ compared with resting NK cells. IL-2 dependency was observed for the secretion of the listed cytokines and NK cell cytolytic function. The results are awaited of a Phase I clinical trial testing adoptive transfer of ex vivo expanded autologous NK cells given every 3 weeks, following treatment with bortezomib and concomitant IL-2, in patients with advanced metastatic tumors and hematological malignancies [51].

\section{Differing effects of bortezomib on T and NK cells}

A study by Lundqvist et al. showed that bortezomib sensitizes tumors to autologous NK cell cytotoxicity as well as enhances the regulatory T-cell population in depleted hosts. Cancer cells treated in vitro with bortezomib had an increased susceptibility to NK cytolysis involving both the TRAIL and perforin/granzyme lytic pathways. In addition, tumor-bearing mice treated with bortezomib and syngeneic NK cells exhibited reduced tumor growth and prolonged survival relative to controls receiving bortezomib or NK cells alone [18]. However, there was no delay in tumor progression after receiving bortezomib and perforin-deficient NK cells suggesting drug-induced enhancement in NK cell cytotoxicity in vivo was mediated via the perforin/ granzyme pathway. Moreover, depletion of Treg cells in the host with anti-CD25 Ab provided an addtional therapeutic benefit [18]. Therefore, Treg cell depletion followed by bortezomib-induced tumor sensitization to autologous NK cell lysis may be another approach for improving NK cell therapies.

Building on these observations, a subsequent study by Lundqvist et al. explored whether the same effects of bortezomib could be used to sensitize tumors to Ag-specific cytotoxic T lymphocytes (CTLs). However, while bortezomib-treated tumors exhibited increased sensitivity to NK cells, they concurrently became resistant to killing by Ag-specific CTLs [52]. The reduced CTL killing lasted for days, but was not due to changes in MHC class-I expression on tumor. It was overcome by pulsing tumors with peptides identified by tumor-reactive CTLs. This was clear from the observation that tumor growth was increased in severe combined immunodeficiency mice when cocultures of tumor-reactive CTLs and bortezomib-treated tumors were injected relative to untreated tumors. In contrast, tumor growth was decreased in mice when cocultures of NK cells and bortezomib-treated tumors were injected relative to untreated tumors. This difference in growth of bortezomib-treated tumors was explained by their sensitization to lysis by NK cells and acquisition of resistance to CTLs, maybe as a result of bortezomib altering the proteasomal processing and presentation of tumor Ags.

Taken together, new therapeutic strategies against cancer can be developed by harnessing the power of depleting Tregs and activating NK cells following bortezomib treatment. The expansion of NK cells using IL-2 cytokine may serve a key strategy to activating NK cells to eradicate tumor cells. Treg cell depletion followed by bortezomib-induced tumor sensitization to autologous NK cells may be used as a beneficial treatment approach for cancer.

\section{Effects of bortezomib treatment in noncancer disease states \\ EBV infections}

Iwata $e t$ al. investigated the effects of bortezomib on both $\mathrm{T}$ and NK cells in an EBV model. EBV is associated with multiple lymphoid malignancies and infects B cells, T cells and NK cells. Although bortezomib was reported to induce apoptosis in EBV-transformed $\mathrm{B}$ cells, bortezomib treatment decreased the viability of multiple T- and NK-cell lymphoma lines, independent of EBV infection by enhanced activation of caspases and polyadenosine diphosphate-ribose polymerase, increased expression of phosphorylated $I \kappa B$ and decreased expression of inhibitor of apoptotic proteins (IAPs) [53]. Administration of bortezomib to patients with EBV-associated lymphoproliferative diseases 
resulted in a greater cyotoxic effect on EBV-infected peripheral blood mononuclear cells.

\section{Inflammatory osteolysis}

The major cause of aseptic loosening following joint replacement is wear particle-induced inflammatory osteolysis [54]. Macrophages are the predominant cell type within periprosthetic tissues. Bortezomib has been investigated for its anti-inflammatory effects on murine macrophage-like RAW 264.7 cells stimulated with titanium particles. In RAW 264.7 cells cultured with titanium particles, the expression of inflammatory cytokines (TNF- $\alpha$, IL-1 $\beta$, IL- 6 and IL-10), chemokines (MCP-1, MIP-1 $\alpha$ ) and inflammatory enzymes (inducible nitric oxide synthase [iNOS], cyclooxygenase-2 [COX-2]) increased. Bortezomib treatment significantly reduced the expression of TNF- $\alpha$, IL-1 $\beta$, IL-6, MCP-1, MIP- $1 \alpha$, iNOS and COX-2 and induced the expression of IL-10 in a timedependent manner [54]. These observations suggest that bortezomib inhibits titanium-induced inflammation in macrophages. Thus, bortezomib treatment could possibly serve as a therapeutic target for preventing or treating aseptic loosening that may be caused by inflammation.

\section{Liver grafts}

Steatotic liver grafts tolerate ischemia-reperfusion (I/R) injury poorly, contributing to increased primary graft dysfunction following transplantation [55]. Activation of NF- $\kappa B$ following I/R injury serves a vital role in activation of pro-inflammatory responses leading to injury. It was evaluated if bortezomib treatment would assist with decreasing pro-inflammatory responses that would reduce I/R injury. To define the mechanisms of steatotic liver injury, the role of NF- $\mathrm{BB}$ was also evaluated by using an orthotopic liver transplant (OLT) model in Zucker rats. Donors treated with bortezomib demonstrated a significant increase in the activation of NF- $\kappa B$, specifically the p 65 subunit following transplantation. Steatotic donor pretreatment with bortezomib resulted in significant reduction in levels of

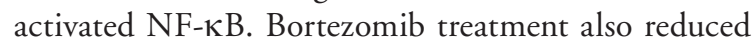
expression of pro-inflammatory cytokines MIP-2, TNF- $\alpha$ and IL-1 $\beta$ along with a significant reduction in liver enzyme ALT levels in obese donors. These observations suggest that I/R injury in steatotic liver transplantation are associated with exaggerated activation of NF- $\mathrm{\kappa B}$ subunit $\mathrm{p} 65$, leading to an inflammatory mechanism of reperfusion injury and necrosis. Bortezomib treatment in steatotic liver donor reduces NF- $\kappa B$ p 65 activation and inflammatory I/R injury, improving transplant outcomes of steatotic grafts in a rat model [55].

\section{Atherosclerosis}

Low and nontoxic proteasome inhibition has antiinflammatory, antiproliferative and antioxidative effects on vascular cells in vitro and in vivo [56]. It is believed that low dose bortezomib treatment could provide antiatherogenic protection. Hence, the effect of bortezomib treatment on early atherosclerotic lesion formation in low-density lipoprotein receptordeficient mice fed a Western-type diet was investigated. Although bortezomib treatment did not affect cholesterol plasma levels, it did significantly reduce atherosclerotic lesion coverage in bortezomib-treated mice. Bortezomib also significantly reduced vascular cellular adhesion molecule-1 expression and macrophage infiltration. There was also a reduction of superoxide content, lipid peroxidation, protein oxidation products, serum levels of MCP-1 and IL-6 following bortezomib treatment. Gene expression microarray analysis also showed that changes induced by Western-type diet were attenuated by bortezomib treatment. Thus, bortezomib treatment exerts antioxidative and anti-inflammatory effects and attenuates development of atherosclerotic lesions in low-density lipoprotein receptor-deficient mice [56].

\section{Graft-versus-host disease}

The pathogenesis of graft-versus-host disease (GVHD) is linked to the chemotactic movement of T-lymphocytes following allogeneic hematopoietic stem cell transplantation [57]. Two chemokine receptors, CCR7 and CXCR3, are associated with the development of GVHD. CCR7 may regulate the development of GVHD by controlling the subpopulation of memory T-cells. CXCR3 helps to function in inflammation and homeostasis. CXCL9, CXCL10, CXCL11 are IFN- $\gamma$-inducible chemokines that bind to CXCR3, to mediate cell migration [58,59]. A 10-nM dose of bortezomib treatment was found to prevent GVHD in a mouse model and to decrease the production of Th1 cytokines such as IFN- $\gamma$ and IL-2. It has been reported that bortezomib differentially regulates the expression of chemokine receptors CXCR3 and CCR7 on the $\mathrm{CD}^{+} / \mathrm{CD}^{+}$subsets of $\mathrm{T}$ cells in a dose-dependent manner [57]. It was seen that increasing concentrations of bortezomib also downregulated the secretion of CXCL9 by activated T cells. Bortezomib inhibited T-cell chemotatic movements toward CXCL9 in a dose-dependent manner, but had no effect on CCL19-induced T-cell chemotaxis. Additionally, bortezomib treatment also triggered T-lymphocyte apoptosis through activation of caspase-3 and its downstream poly-ADP-ribose polymerase cleavage in a dose- and time-dependent manner [57]. Bortezomib suppresses acute lethal GVHD response in myeloablated mice following allogenic bone marrow transfer when multiple 
injections of bortezomib close to the maximal tolerated levels were administered [60]. Thus, bortezomib may suppress GVHD by downregulating T-cell chemotatic movement toward GVHD target organs and by inducing T-cell apoptosis at an optimal treatment regimen.

\section{Colitis}

Chronic inflammation is a characteristic feature of inflammatory bowel disease (IBD), comprising Crohn's disease and ulcerative colitis, in the gut [61]. Increased proteasome activity was found to enhance proinflammatory signaling and thus promote inflammation in patients with IBD. A study was designed to address whether modulation of the proteasomal activity is a suitable therapeutic approach to limit inflammation in colitis. Development of dextran sodium sulfate induced colitis was tested in mice treated with bortezomib. Modulation of the proteasome activity by bortezomib limited the secretion of proinflammatory cytokines and chemokines such as IL-6, IL-17, MIP-1 $\alpha$, MIP-2 and neutrophil attractant $\mathrm{KC}$ in dextran sodium sulfate induced colitis. The results revealed that reduction of the proteasome activity by bortezomib may be developed as a suitable treatment modality to attenuate inflammation in gastro-intestinal diseases [61].

\section{Toxic shock syndrome}

Although bortezomib can decrease the production of proinflammatory cytokines and chemokines by inhibiting the NF- $\mathrm{BB}$ inflammation pathway, this effect can be detrimental in conditions of acute systemic inflammation [62]. The therapeutic and prophylactic use of bortezomib in bacterial super Ag (BSAg) induced toxic shock syndrome and bacterial LPSinduced shock were evaluated using murine models. Prophylactic bortezomib significantly reduced serum levels of many cytokines and chemokines induced by BSAg such as: IL-1 $\alpha$, IL-3, IL-4, IL-5, IL-6, IL12p40, IL-12p70, IL-13, IFN- $\gamma$, TNF- $\alpha$, MIP-1 $\beta$, GM-CSF, CXCL1, RANTES and Eotaxin. These effects were most pronounced at $3 \mathrm{~h}$ after bortezomib treatment, with no observable effects on TNF- $\alpha$ at $6 \mathrm{~h}$. Bortezomib treatment also significantly reduced the NF- $\kappa B$ transcription factors $\mathrm{p} 65$ and $\mathrm{p} 50$ in the liver tissue from staphylococcal enterotoxin $\mathrm{B}$ challenged mice [62]. Because NF- $\kappa \mathrm{B}$-dependent antiapoptotic pathways protect hepatocytes from TNF$\alpha$-induced cell death, inhibition of NF- $\kappa \mathrm{B}$ as a result of the bortezomib treatment, in the face of elevated TNF- $\alpha$ levels caused by BSAg or LPS, could increase toxicity due to a sensitization of target organs to death ligand-mediated cytotoxicity [62].

Based on the above studies, it is evident that bortezomib can be used in a variety of disease states to assist with the modulation of cytokines and chemokines, to alleviate the immune response and reduce inflammation in multiple compartments of the body. The most common cytokines and chemokines that were modulated by bortezomib treatment were IL-1 $\beta$, IL-6, IL-12, IFN- $\gamma$, TNF- $\alpha$, MIP-1 $\beta$ and MCP-1. Bortezomib was also found to reverse diminished IL-12-induced IFN-g production by immune cells as a result of reduction in STAT4 protein half-life following chemotherapy [63]. In an inflammatory state, bortezomib is known to inhibit the NF- $\mathrm{KB}$ activation by downregulating NF- $\kappa \mathrm{B}$ transcription factors such as $\mathrm{p} 65$ and $\mathrm{p} 50$. In a cancer state, bortezomib was seen to upregulate proteins such as Bad, p53, p21 and p27 to regulate mitochondrial control of apoptosis, tumor suppression and the cell cycle of tumor cells, as well as ameliorate T-cell and/or NK cell effector responses against tumor cells. It is thus evident that bortezomib can be used in many disease states as a therapeutic strategy.

\section{Conclusion \& future perspective}

\section{Balancing the immune suppressive}

$\&$ stimulatory effects of bortezomib for

efficient immunotherapy

It is intriguing that bortezomib has been shown to have both immunosuppressive and immunostimulatory effects. There are many modulatory roles of bortezomib involving a wide variety of cellular subsets, tumor-associated ligands and lymphocyte activating receptors, cytokine/chemokine signaling and their downstream signaling pathways. Additionally, there are multiple positive and negative effects of bortezomib on different immune cells as summarized in Table 1.

Bortezomib has been observed to play a role in the apoptosis of Ab-secreting B cells [31]. In addition, bortezomib has shown a wide variety of negative effects on DCs. Plasmacytoid DCs were found to be the most susceptible to the killing effects of bortezomib [37,39]. However, it has been demonstrated that by exposing heat shock proteins on dying tumor cells to DCs or by activating chymase 1 gene, which is a chymotryptic serine proteinase that may influence antigen processing and presentation, bortezomib can induce immunity to tumors $[21,64]$. Also, bortezomib can sensitize cancer cells to NK cell mediated killing by enhancing NK cell activating receptors and ligands $[17,46,51]$, and/ or reverse their proliferative and antiapoptotic traits by modulating NF- $\mathrm{BB}$ signaling [65], apoptotic machinery [66], or Notch signaling [67,68]. Similarly, in patients with HCC, bortezomib treatment enhances NKG2Dmediated antitumor effects [47]. Bortezomib also sensitizes CML or ALL cells to NK-mediated lysis $[17,48]$. Also, bortezomib augments tumor cell apoptosis via the inhibition of the cell survival pathways, in particu- 
Table 1. Immunosuppressive and immunostimulatory effects of bortezomib on immune cells. Negative immunosuppressive effects of bortezomib

T cells:

- Decrease in CD4 T-cell count

- Lessened CD8 T-cell cytotoxicity in vitro

B cells:

- Impaired antibody-mediated responses by enhanced B-cell apoptosis

DCs:

- Desensitized DCs to immunostimulation by TNF- $\alpha$ and LPS

- Loss of antigen presentation

- Induction of apoptosis in dendritic cells

- Reduction of DC-induced allogenic T-cell proliferation

- Inhibition of the expression of DC-maturation markers

Positive immunostimulatory effects of bortezomib

DCs:

- Exposure of heat shock protein 90 on DCs to induce immunity to human myeloma

T- and/or NK-cell effector responses:

- Sensitization of various cancer cells to NK cell-mediated lysis

- Greater antitumor effects following treatment with a combination of bortezomib and adoptively transferred activated NK cells

- Stimulation of cytotoxicity and IFN- $\gamma$ production by NK cells cocultured with tumor cells

- Increased TRAIL and perforin/granzyme-mediated caspase-8 activity against tumors

- Reduced tumor growth and prolonged host survival following treatment with bortezomib and syngeneic NK cells

- Ameliorate T-cell and/or NK-cell effector responses against tumor cells

NK-cell-activating receptors and ligands:

- Increased expression of NKG2DL, DNAM-1 and MIC A/B ligands following treatment with

bortezomib at concentrations $<10 \mathrm{nM}$

Cytokine combinatorial treatment on NK cells:

- Bortezomib and IL-12 combination therapy enhances NK-cell antitumor effect

Cytokine/chemokine signaling:

- Reduced inflammatory IL-1 $\beta$, IL-6, IL-12, IFN- $\gamma$, TNF- $\alpha$, MIP-1 $\beta$ and MCP-1 cytokines and chemokines

- Reduces chemotherapy-induced STAT-4 deficiency and IFN- $\gamma$ production

DC: Dendritic cell; NK: Natural killer.

lar by abrogating the constitutive activation of NF- $\mathrm{KB}$ that confers cell death resistance in tumor cells. Bortezomib can improve or overcome chemotherapyinduced STAT4 deficiency [63]. Additionally, in regard to NK cells, combination of bortezomib and cytokines, particularly IL-2 and IL-12, has an extensive antitumor outcome relative to monotherapy of the individual agents. One side effect of bortezomib was an increase in the CD4 Treg population [18]. Therefore, Treg cell depletion combined with bortezomib-induced tumor sensitization to cytotoxic T and/or NK cells may serve a better treatment approach for cancer [18]. Further- more, bortezomib caused significantly reduced serum levels of many proinflammatory and angiogenesis inducible cytokines and chemokines such as: IL- $1 \alpha / \beta$, IL-3，IL-4，IL-5，IL-6, IL-8，IL-12p40，IL-12p70, IL-13, IFN- $\gamma$, TNF- $\alpha$, MIP-1 $\alpha / \beta$, GM-CSF, CXCL1, RANTES, VEGF and Eotaxin [62].

Understanding the diverse contradictory effects of bortezomib requires a consideration of the concentrations of bortezomib that have been used in these various studies. In essence, most experiments that indicate immunosuppressive effects tend to use high concentrations of bortezomib. The test depletion of alloreactive 
T cells with the aim of eliminating GVHD utilized bortezomib concentrations in the range of 100-1000 $\mathrm{nM}$ [69]. In contrast, beneficial effects of bortezomib on immune function that have been shown are mostly concentrations of $20 \mathrm{nM}$ or lower. The level of proteasome inhibition using bortezomib concentrations at $10-20 \mathrm{nM}$ are well tolerated in both mice and humans without appreciable toxicity [26,70-71]. Our recent data are consistent with the fact that bortezomib at lower concentrations can stimulate the production of several immunostimulatory cytokines and chemokines in treated mice and can be beneficial in enhancing the adoptive cell therapy of solid tumors. Thus, the combination of low-dose bortezomib and cytokine treatment, for example, IL-2 or IL-12, along with adoptive transfer of immune cells such as cytotoxic T cells and/ or NK cells may prove to be the optimal strategy in the context of immunotherapy. It is an additional benefit that bortezomib can sensitize cancer cells to immunemediated destruction.

Second-generation proteasome inhibitors, such as carfilzomib [72], recently identified as potent novel sensitizers of cancer cells to apoptosis, which exhibit minimal toxic effects as single agents, might be even more therapeutically advantageous in combination with cytokine and adoptive cell therapy. The therapeutic administration of carefully optimized, minimal dose of proteasome inhibitors could prove effective in providing immune benefits while limiting the negative immunosuppressive effects. More research must be conducted to fully understand the range, safety and timing of these therapeutic combinations, since little is known of their effect on host immune responses.

\section{Acknowledgements}

The authors would like to thank K Muhammad and S Pulliam for their assistance with the critical review of this article.

\section{Disclaimer}

The content of this publication does not necessarily reflect the views or policies of the Department of Health and Human Services, nor does mention of trade names, commercial products, or organizations imply endorsement by the US Government.

\section{Financial \& competing interests disclosure}

A Shanker has been supported by NIH grants U54 CA163069, P50 CA 090949, SC1 CA182843, U54 MD007593 and R01 CA175370. ST Pellom is supported by $\mathrm{NIH}$ training grants T32 5T32HL007737 and R25 GM059994. TJ Sayers has been funded in whole or in part with federal funds from the National Cancer Institute, NIH, under contracts N01-CO-12400 and HHSN261200800001E. This Research was also supported (in part) by the Intramural Research Program of $\mathrm{NIH}$, Frederick National Lab, Center for Cancer Research. The authors have no other relevant affiliations or financial involvement with any organization or entity with a financial interest in or financial conflict with the subject matter or materials discussed in the manuscript apart from those disclosed.

No writing assistance was utilized in the production of this manuscript.

Executive summary

Modulatory effects of bortezomib on host immune cell functions

- Proteasome inhibition by bortezomib has both immunosuppressive and immunostimulatory effects.

- There are many modulatory roles of bortezomib involving a wide variety of cellular subsets, tumor-associated ligands and lymphocyte activating receptors, cytokine/chemokine signaling and their downstream signaling pathways.

- Bortezomib plays a role in the apoptosis of antibody-secreting B cells.

- Plasmacytoid dendritic cells are very susceptible to the killing effects of bortezomib.

- After exposing heat shock proteins on dying tumor cells, bortezomib can improve antigen presentation by dendritic cells and elicit immunity to tumors.

- Bortezomib can sensitize cancer cells to natural killer (NK)-cell-mediated killing by enhancing expression of NK cell-activating receptors and ligands.

- Bortezomib treatment enhances NKG2D-mediated antitumor effects.

- Bortezomib augments tumor cell apoptosis via the inhibition of the cell survival pathways.

- Combination of bortezomib and cytokine treatments with adoptive cell transfer of T cells and/or NK cells is expected to have extensive antitumor outcome.

- A side effect of bortezomib is an increase in the CD4 T-regulatory population.

- Bortezomib decreases serum levels of many proinflammatory and angiogenesis-inducing cytokines and chemokines.

- Experiments that indicate immunosuppressive effects of bortezomib tend to use high concentrations in the range of $100-1000 \mathrm{nM}$.

- Beneficial effects of bortezomib on immune function are mostly at concentrations of $20 \mathrm{nM}$ or lower.

- The therapeutic administration of carefully optimized, minimal bortezomib doses could prove effective in providing immune benefits while limiting the negative immunosuppressive effects. 


\section{References}

Papers of special note have been highlighted as:

- of interest; $\bullet \bullet$ of considerable interest

1 Pellom ST Jr, Shanker A. Development of proteasome inhibitors as therapeutic drugs. J. Clin. Cell Immunol. S5, 5 (2012).

2 Ciechanover A. Proteolysis: from the lysosome to ubiquitin and the proteasome. Nat. Rev. Mol. Cell Biol. 6(1), 79-87 (2005)

3 Hershko A, Ciechanover A. The ubiquitin system. Annu. Rev. Biochem. 67, 425-479 (1998).

4 Xie Y. Structure, assembly and homeostatic regulation of the 26s proteasome. J. Mol. Cell Biol. 2(6), 308-317 (2010).

5 Driscoll JJ, Dechowdhury R. Therapeutically targeting the sumoylation, ubiquitination and proteasome pathways as a novel anticancer strategy. Target Oncol. 5(4), 281-289 (2010).

6 Li T, Ho L, Piperdi B et al. Phase II study of the proteasome inhibitor bortezomib (ps-341, velcade) in chemotherapynaive patients with advanced stage non-small cell lung cancer (NSCLC). Lung Cancer 68(1), 89-93 (2010).

7 O'Connor OA, Wright J, Moskowitz C et al. Phase II clinical experience with the novel proteasome inhibitor bortezomib in patients with indolent non-hodgkin's lymphoma and mantle cell lymphoma. J. Clin. Oncol. 23(4), 676-684 (2005).

8 Berges C, Haberstock H, Fuchs D et al. Proteasome inhibition suppresses essential immune functions of human $\mathrm{CD}^{+}{ }^{+} \mathrm{T}$ cells. Immunology 124(2), 234-246 (2008).

9 Feng X, Yan J, Wang Y et al. The proteasome inhibitor bortezomib disrupts tumor necrosis factor-related apoptosisinducing ligand (TRAIL) expression and natural killer (NK) cell killing of TRAIL receptor-positive multiple myeloma cells. Mol. Immunol. 47(14), 2388-2396 (2010).

10 Heider U, Rademacher J, Kaiser M, Kleeberg L, Von Metzler $\mathrm{I}$, Sezer O. Decrease in $\mathrm{CD} 4^{+} \mathrm{T}$-cell counts in patients with multiple myeloma treated with bortezomib. Clin. Lymphoma Myeloma Leuk. 10(2), 134-137 (2010).

11 Straube C, Wehner R, Wendisch M et al. Bortezomib significantly impairs the immunostimulatory capacity of human myeloid blood dendritic cells. Leukemia 21(7), 1464-1471 (2007).

- Demonstration of the impairment of the antigen-presenting dendritic cells by bortezomib.

12 Zinser E, Rossner S, Littmann L, Luftenegger D, Schubert $\mathrm{U}$, Steinkasserer A. Inhibition of the proteasome influences murine and human dendritic cell development in vitro and in vivo. Immunobiology 214(9-10), 843-851 (2009).

13 Mulder A, Heidt S, Vergunst M, Roelen DL, Claas FH. Proteasome inhibition profoundly affects activated human B cells. Transplantation 95(11), 1331-1337 (2013).

14 Ames E, Hallett WH, Murphy WJ. Sensitization of human breast cancer cells to natural killer cell-mediated cytotoxicity by proteasome inhibition. Clin. Exp. Immunol. 155(3), 504-513 (2009)

- Study of bortezomib's effect on natural killer (NK)-cellmediated cytotoxicity against cancer cells.
15 Armeanu S, Krusch M, Baltz KM et al. Direct and natural killer cell-mediated antitumor effects of low-dose bortezomib in hepatocellular carcinoma. Clin. Cancer Res. 14(11), 3520-3528 (2008).

16 Chang CL, Hsu YT, Wu CC et al. Immune mechanism of the antitumor effects generated by bortezomib. J. Immunol. 189(6), 3209-3220 (2012).

17 Hallett WH, Ames E, Motarjemi M et al. Sensitization of tumor cells to NK cell-mediated killing by proteasome inhibition. J. Immunol. 180(1), 163-170 (2008).

- First study of sensitization of tumor cells to NK-cell killing by bortezomib.

18 Lundqvist A, Yokoyama H, Smith A, Berg M, Childs R. Bortezomib treatment and regulatory $\mathrm{T}$-cell depletion enhance the antitumor effects of adoptively infused NK cells. Blood 113(24), 6120-6127 (2009).

-. Initial demonstration of the benefits of combinatorial bortezomib treatment with $\mathrm{T}$-cell depletion and adoptive NK-cell transfer.

19 Seeger JM, Schmidt P, Brinkmann K et al. The proteasome inhibitor bortezomib sensitizes melanoma cells toward adoptive CTL attack. Cancer Res. 70(5), 1825-1834 (2010).

20 Shi J, Tricot GJ, Garg TK et al. Bortezomib down-regulates the cell-surface expression of HLA class I and enhances natural killer cell-mediated lysis of myeloma. Blood 111(3), 1309-1317 (2008).

21 Spisek R, Charalambous A, Mazumder A, Vesole DH, Jagannath S, Dhodapkar MV. Bortezomib enhances dendritic cell (DC)-mediated induction of immunity to human myeloma via exposure of cell surface heat shock protein 90 on dying tumor cells: therapeutic implications. Blood 109(11), 4839-4845 (2007).

22 Jagannath S, Barlogie B, Berenson J et al. A Phase 2 study of two doses of bortezomib in relapsed or refractory myeloma. Br. J. Haematol. 127(2), 165-172 (2004).

23 Chanan-Khan A, Sonneveld P, Schuster MW et al. Analysis of herpes zoster events among bortezomib-treated patients in the Phase III apex study. J. Clin. Oncol. 26(29), 4784-4790 (2008)

24 Blanco B, Perez-Simon JA, Sanchez-Abarca LI et al. Bortezomib induces selective depletion of alloreactive $\mathrm{T}$ lymphocytes and decreases the production of Th1 cytokines. Blood 107(9), 3575-3583 (2006).

25 Blanco B, Perez-Simon JA, Sanchez-Abarca LI et al. Treatment with bortezomib of human $\mathrm{CD}^{+}{ }^{+} \mathrm{T}$ cells preserves natural regulatory $\mathrm{T}$ cells and allows the emergence of a distinct suppressor T-cell population. Haematologica 94(7), 975-983 (2009).

26 Shanker A, Brooks AD, Tristan CA et al. Treating metastatic solid tumors with bortezomib and a tumor necrosis factorrelated apoptosis-inducing ligand receptor agonist antibody. J. Natl Cancer Inst. 100(9), 649-662 (2008).

-• First demonstration of the efficacy of bortezomib treatment in murine solid tumors.

27 Kim JE, Jin DH, Lee WJ, Hur D, Wu TC, Kim D. Bortezomib enhances antigen-specific cytotoxic $\mathrm{T}$ cell responses against immune-resistant cancer cells generated 
by STAT3-ablated dendritic cells. Pharmacol. Res. 71, 23-33 (2013).

Jazirehi AR, Economou JS. Proteasome inhibition blocks NF-kappaB and Erk1/2 pathways, restores antigen expression, and sensitizes resistant human melanoma to TCR-engineered CTLs. Mol. Cancer Ther. 11(6), 1332-1341 (2012).

29 Hideshima T, Ikeda H, Chauhan D et al. Bortezomib induces canonical nuclear factor-kappaB activation in multiple myeloma cells. Blood 114(5), 1046-1052 (2009).

30 Cenci S, Mezghrani A, Cascio P et al. Progressively impaired proteasomal capacity during terminal plasma cell differentiation. EMBO J. 25(5), 1104-1113 (2006).

31 Cascio P, Oliva L, Cerruti F et al. Dampening Ab responses using proteasome inhibitors following in vivo $\mathrm{B}$ cell activation. Eur. J. Immunol. 38(3), 658-667 (2008).

32 Schakel K, Kannagi R, Kniep B et al. 6-sulfo lacnac, a novel carbohydrate modification of PSGL-1, defines an inflammatory type of human dendritic cells. Immunity 17(3), 289-301 (2002).

33 Schakel K, Mayer E, Federle C, Schmitz M, Riethmuller G, Rieber EP. A novel dendritic cell population in human blood: one-step immunomagnetic isolation by a specific $\mathrm{mAb}(\mathrm{m}$ DC8) and in vitro priming of cytotoxic T lymphocytes. Eur. J. Immunol. 28(12), 4084-4093 (1998).

34 Schakel K, Von Kietzell M, Hansel A et al. Human 6-sulfo lacnac-expressing dendritic cells are principal producers of early interleukin-12 and are controlled by erythrocytes. Immunity 24(6), 767-777 (2006).

35 Radtke F, Macdonald HR, Tacchini-Cottier F. Regulation of innate and adaptive immunity by Notch. Nat. Rev Immunol. 13(6), 427-437 (2013).

36 Auderset F, Coutaz M, Tacchini-Cottier F. The role of Notch in the differentiation of $\mathrm{CD} 4\left(^{+}\right) \mathrm{T}$ helper cells. Curr. Top. Microbiol. Immunol. 360, 115-134 (2012).

37 Amsen D, Blander JM, Lee GR, Tanigaki K, Honjo T, Flavell RA. Instruction of distinct CD4 T helper cell fates by different Notch ligands on antigen-presenting cells. Cell 117(4), 515-526 (2004).

38 Ogawa Y, Tobinai K, Ogura M et al. Phase I and II pharmacokinetic and pharmacodynamic study of the proteasome inhibitor bortezomib in japanese patients with relapsed or refractory multiple myeloma. Cancer Sci. 99(1), 140-144 (2008).

39 Amsen D, Antov A, Flavell RA. The different faces of Notch in T-helper-cell differentiation. Nat. Rev. Immunol. 9(2), 116-124 (2009).

40 Iwakoshi NN, Lee AH, Vallabhajosyula P, Otipoby KL, Rajewsky K, Glimcher LH. Plasma cell differentiation and the unfolded protein response intersect at the transcription factor XBP-1. Nat. Immunol. 4(4), 321-329 (2003).

41 Tanaka S, Tsukada J, Suzuki W et al. The interleukin-4 enhancer CNS-2 is regulated by Notch signals and controls initial expression in NKT cells and memory-type CD4 T cells. Immunity 24(6), 689-701 (2006).

42 Subklewe M, Sebelin-Wulf K, Beier C et al. Dendritic cell maturation stage determines susceptibility to the proteasome inhibitor bortezomib. Hum. Immunol. 68(3), 147-155 (2007).

43 Hu W, Zheng RR, Cui HX, Yue D, Wang Y, Jiang YH. Effects of bortezomib in sensitizing human prostate cancer cell lines to NK-mediated cytotoxicity. Asian J. Androl 14(5), 695-702 (2012).

44 Yong AS, Keyvanfar K, Hensel N et al. Primitive quiescent $\mathrm{CD} 34^{+}$cells in chronic myeloid leukemia are targeted by in vitro expanded natural killer cells, which are functionally enhanced by bortezomib. Blood 113(4), 875-882 (2009).

45 Bosman MC, Schuringa JJ, Quax WW, Vellenga E. Bortezomib sensitivity of AML CD34 ${ }^{+}$cells can be enhanced by targeting the persisting activity of NF-kB and the accumulation of MCL-1. Exp. Hematol. 41(6), 530-538 (2013).

46 Soriani A, Zingoni A, Cerboni C et al. ATM-ATRdependent up-regulation of DNAM-1 and NKG2D ligands on multiple myeloma cells by therapeutic agents results in enhanced NK-cell susceptibility and is associated with a senescent phenotype. Blood 113(15), 3503-3511 (2009).

47 Butler JE, Moore MB, Presnell SR, Chan HW, Chalupny NJ, Lutz CT. Proteasome regulation of ULBP1 transcription. J. Immunol. 182(10), 6600-6609 (2009).

48 Jardine L, Hambleton S, Bigley V, Pagan S, Wang XN, Collin M. Sensitizing primary acute lymphoblastic leukemia to natural killer cell recognition by induction of NKG2D ligands. Leuk. Lymphoma 54(1), 167-173 (2013).

49 Khan T, Stauffer JK, Williams R et al. Proteasome inhibition to maximize the apoptotic potential of cytokine therapy for murine neuroblastoma tumors. J. Immunol. 176(10), 6302-6312 (2006).

50 Wang X, Feng X, Wang J et al. Bortezomib and IL12 produce synergetic anti-multiple myeloma effects with reduced toxicity to natural killer cells. Anticancer Drugs 25(3), 282-288 (2014).

51 Berg M, Lundqvist A, Mccoy P Jr et al. Clinical-grade ex vivo-expanded human natural killer cells up-regulate activating receptors and death receptor ligands and have enhanced cytolytic activity against tumor cells. Cytotherapy 11(3), 341-355 (2009).

- Important study investigating bortezomib treatment and ex vivo expanded NK-cell transfer to combat tumor cells.

52 Lundqvist A, Su S, Rao S, Childs R. Cutting edge: Bortezomib-treated tumors sensitized to NK cell apoptosis paradoxically acquire resistance to antigen-specific $\mathrm{T}$ cells. J. Immunol. 184(3), 1139-1142 (2010).

53 Iwata S, Yano S, Ito Y et al. Bortezomib induces apoptosis in T lymphoma cells and natural killer lymphoma cells independent of Epstein-Barr virus infection. Int. J. Cancer 129(9), 2263-2273 (2011).

54 Mao X, Pan X, Peng X, Cheng T, Zhang X. Inhibition of titanium particle-induced inflammation by the proteasome inhibitor bortezomib in murine macrophage-like RAW 264.7 cells. Inflammation 35(4), 1411-1418 (2012).

55 Ramachandran S, Liaw JM, Jia J et al. Ischemia-reperfusion injury in rat steatotic liver is dependent on NFkappaB p 65 activation. Transpl. Immunol. 26(4), 201-206 (2012). 
56 Wilck N, Fechner M, Dreger $\mathrm{H}$ et al. Attenuation of early atherogenesis in low-density lipoprotein receptor-deficient mice by proteasome inhibition. Arterioscler. Thromb. Vasc. Biol. 32(6), 1418-1426 (2012).

57 Liu W, Ren HY, Dong YJ et al. Bortezomib regulates the chemotactic characteristics of $\mathrm{T}$ cells through downregulation of CXCR3/CXCL9 expression and induction of apoptosis. Int. J. Hematol. 96(6), 764-772 (2012).

58 Broxmeyer HE. Chemokines and chemokine receptors in hematopoiesis and immunology. Exp. Hematol. 34(8), 965-966 (2006).

59 Moser B, Loetscher P. Lymphocyte traffic control by chemokines. Nat. Immunol. 2(2), 123-128 (2001).

60 Sun K, Welniak LA, Panoskaltsis-Mortari A et al. Inhibition of acute graft-versus-host disease with retention of graftversus-tumor effects by the proteasome inhibitor bortezomib. Proc. Natl Acad. Sci. USA 101(21), 8120-8125 (2004).

61 Schmidt N, Gonzalez E, Visekruna A et al. Targeting the proteasome: partial inhibition of the proteasome by bortezomib or deletion of the immunosubunit LMP7 attenuates experimental colitis. Gut 59(7), 896-906 (2010).

62 Tilahun AY, Theuer JE, Patel R, David CS, Rajagopalan G. Detrimental effect of the proteasome inhibitor, bortezomib in bacterial superantigen- and lipopolysaccharide-induced systemic inflammation. Mol. Ther. 18(6), 1143-1154 (2010).

63 Lupov IP, Voiles L, Han L et al. Acquired STAT4 deficiency as a consequence of cancer chemotherapy. Blood 118(23), 6097-6106 (2011).

64 Liao ZJ, Ma WL, Meng W, Liang S, Meng FY, Zheng WL. Effect of velcade on the gene expression profiles of K562 cells: Study of its molecular mechanism. Nan Fang Yi Ke Da Xиe Xue Bao 28(3), 373-376 (2008).
65 Tsapakidis K, Vlachostergios PJ, Voutsadakis IA et al. Bortezomib reverses the proliferative and antiapoptotic effect of neuropeptides on prostate cancer cells. Int. J. Urol. 19(6), 565-574 (2012).

66 Shanker A, Sayers T. Sensitizing tumor cells to immunemediated cytotoxicity. Adv. Exp. Med. Biol. 601, 163-171 (2007).

67 Chen F, Pisklakova A, Li M, Baz R, Sullivan DM, Nefedova Y. Gamma-secretase inhibitor enhances the cytotoxic effect of bortezomib in multiple myeloma. Cell Oncol. (Dordr.) 34(6), 545-551 (2011).

$68 \mathrm{Xu} \mathrm{D}, \mathrm{Hu} J, \mathrm{Xu} \mathrm{S}$ et al. DLL1/Notch activation accelerates multiple myeloma disease development by promoting CD138+ MM-cell proliferation. Leukemia 26(6), 1402-1405 (2012).

69 Blanco B, Sanchez-Abarca LI, Caballero-Velazquez T, Santamaria C, Inoges S, Perez-Simon JA. Depletion of alloreactive T-cells in vitro using the proteasome inhibitor bortezomib preserves the immune response against pathogens. Leuk. Res. 35(10), 1412-1415 (2011).

70 Adams J, Palombella VJ, Sausville EA et al. Proteasome inhibitors: a novel class of potent and effective antitumor agents. Cancer Res. 59(11), 2615-2622 (1999).

-. The original publication showing the efficacy of the proteasome inhibitor bortezomib as an anticancer agent.

71 Lightcap ES, Mccormack TA, Pien CS, Chau V, Adams J, Elliott PJ. Proteasome inhibition measurements: clinical application. Clin. Chem. 46(5), 673-683 (2000).

72 Arastu-Kapur S, Anderl JL, Kraus M et al. Nonproteasomal targets of the proteasome inhibitors bortezomib and carfilzomib: a link to clinical adverse events. Clin. Cancer Res. 17(9), 2734-2743 (2011). 